\title{
Analysis of Robotic Vision by Using Image Processing Trying to Find the Shortest Path to Goal Using Voronoi Diagram
}

\author{
Amani Y. Noori and Suhad F. Majeed \\ Department of Computer science, College of Basic Education, University of Mustansiriyah. \\ E-mail: snowhite105@gmail.com \\ E-mail: soledad.ahmed@yahoo.com
}

\begin{abstract}
In this paper we present a method to navigate a mobile robot for finding the path to his goal. This method determines the shortest path for robot to transverse to its target location while detecting and avoiding obstacles of different shapes in indoor environment. The vision of robot is first captured as an image using laptop cam, the image analysis is based on image processing in Matlab to identify the existence of obstacles within the environment.

Voronoi diagrams (VDS) is a roadmap method that provides all possible paths in an environment containing stationary obstacles. By using a distance formula we get the shortest path for robot among three paths.

The program is written in Matlab 2010 with image processing toolbox, the proposed technique does not make use of any other type of sensor other than laptop cam.
\end{abstract}

[DOI: $10.22401 / \mathrm{JNUS} .20 .1 .19]$

Keywords: mobile robot, obstacles, voronoi diagrams, path planning, morphological operation.

\section{Introduction}

Image processing is a form of signal processing where the input signals are images such as photographs or video frames. The output could be a transformed version of the input image or a set of characteristics or parameters related to the image. The computer revolution that has taken place over the last 20 years has led to great advancements in the field of digital image processing. This has in turn, opened up a multitude of applications in various fields, in which the technology could be utilized [1][2].

This paper present a method of vision based on robot navigation, it has long been a fundamental goal in both robotics and computer vision research. For a mobile robot to navigate successfully to a goal whilst avoiding both static and dynamic obstacles is a challenging problem. While the problem is largely solved for robots equipped with active range-finding devices, for a variety of reasons, the task still remains challenging for robots equipped only with vision sensors.

Vision is an attractive sensor as it helps in the design of economically viable systems with simpler sensor limitations.

It facilitates passive sensing of the environment and provides valuable semantic information about the scene that is unavailable to other sensors. Several different approaches to avoiding obstacles have been developed in recent years, some of which are computationally intensive. With the help of a laptop camera, a path can be generated for the robot by capturing and processing real time images [3]. Akihisa Ohya, describe a vision based on navigation method in an indoor environment for an autonomous mobile robot which can avoid obstacles. In this method, the self - localization of the robot is done with a model-based vision system and nonstop navigation is realized by a retroactive position correction system, Stationary obstacles are avoided with single-camera.

Vision and moving obstacles are detected with ultrasonic sensors [4].

Buniyamin N., present an overview of path planning algorithms for autonomous robots. The paper then focuses on the bug algorithm family which is a local path planning algorithm. Bug algorithms use sensors to detect the nearest obstacle as a mobile robot moves towards a target with limited information about the environment. The algorithm uses obstacle border as guidance toward the target as the robot circum navigates the obstacle till it finds certain condition to 
fulfill the algorithm criteria to leave the obstacle toward target point [5].

Abhishek Chandaket, present a wavefront based method to create a path for a robot while detecting and avoiding obstacles of different shapes in indoor environment overhead camera is used to acquire an image of environment .The image analysis is based on general processing in Matlab. On that basis, 4 point and 8 point connectivity based algorithms are presented for the purpose of path generation. But this technique may not always give the shortest path for navigation increasing the area of operation is costly as the processing time and complexity increases with increase in the number of cameras used to capture the image. This technique is tested for indoor environment only [3].

\section{System setting and environment}

Autonomous robot which work without human operators are required in robotic fields. Nowadays, these mobile robots have to be equipped with a capability to navigate in environment to execute a given task while avoiding obstacles. A number of sensors such as laser finder ultrasonic sensors, and etc are used widely in order to detect obstacles in environment. However most of these sensors are too expensive. Hence, Visual navigation took much attention after web cameras were introduced a few years ago their cost is attractive as compared To the previous sensors, In order to achieve tasks, It is necessary to determine an optimal or feasible path for the robot when it moves from start point to target point while avoiding obstacles in its way [3]. Visual servo is the use of image data in closed loop control of a robot. Without doubt, today, the use of vision in robotic applications is rapidly increasing.

This is due to the fact that vision based sensors such as webcams are falling in price more rapidly than any other sensor. It is also a richer sensor than traditional ranging devices, particularly since a camera captures much more data simultaneously [6]. In this paper didn't used real robot it present a method for visual servo using only visual images to describe a physical simulation for vision of robot to determine all possible paths through stationary obstacles in indoor environment.
By using Image processing, analysis capture image from laptop cam. We used an approach based on the Voronoi diagram as a roadmap for detect the polygons obstacles in an environment. Once this diagram has been constructed, we can determine the shortest path for the robot among all possible paths.

\section{Methodology}

The path to be transverse by the robot must be ensured to be free of obstacles. For this vision of the robot is representing as an image capture by the laptop camera and analysis by using image processing tools in Matlab. Basic morphological operations are performed on this image to get rid of the noise and unnecessary details. The final image so obtained is used to determine if there is any obstacle in the environment.

The block diagram bellow is given to describe the methodology of this paper. 


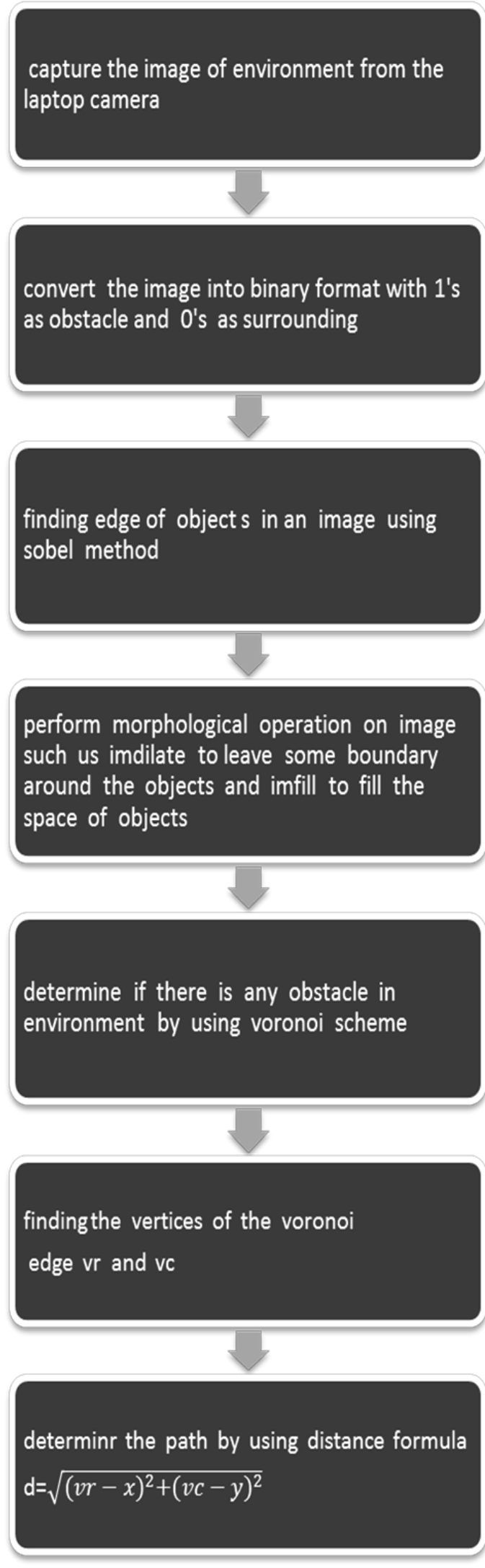

Fig.(1): Block diagram of methodology.

The following steps to express each operation:

1) Generate input video objects This can be implemented using the command
Obj = videoinput ('adaptorname', devicename, 'format');

Where the adaptorname can be determined using the 'imaqhwinfo' command device name is the name given to the device and format refers to the required image format.

- Preview the video

Preview (obj);

Where obj is in the last command

Set brightness level of image. (optional)

set (obj, 'property name', property value);

- Capture image from video

$\mathrm{a}=$ getsnapshot (obj);

The capture image is stored as an array whose elements represent the light level as shown in Fig.(2).

- Remove the input device from memory delete (obj);

2) convert image from RGB to binary image as shown in Fig.(3)

$\mathrm{q} 1=\operatorname{rgb} 2$ gray $(\mathrm{a})$;

$\mathrm{q} 2=\mathrm{im} 2 \mathrm{bw}(\mathrm{q} 1)$;

3) Find edges of objects in the image by using sobel method as shown in Fig.(4). e1 = edge ( q2, ' sobel ' ) ;

Where edge detection is an image processing technique for finding the boundaries of objects in an image.

It's used for data extraction in areas such as image processing, computer vision, and machine vision. Common edge detection methods include Sobel, Canny, Prewitt, and fuzzy logic methods.

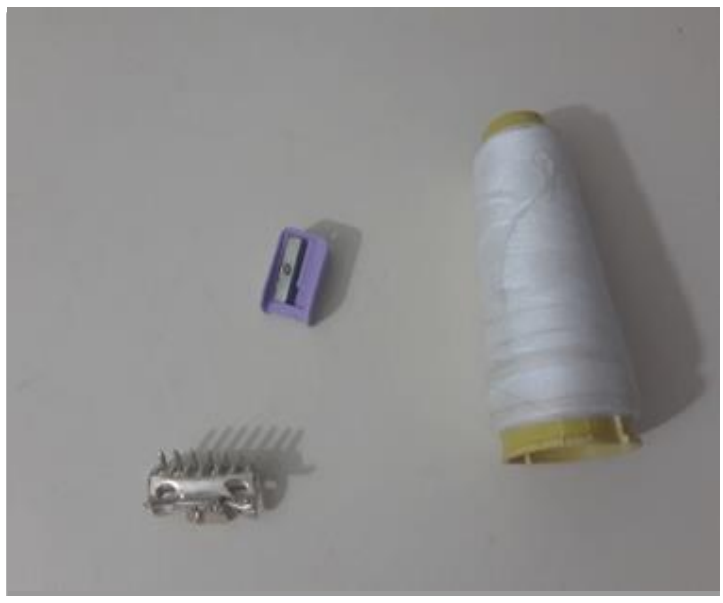

Fig.(2): Capture image from video. 


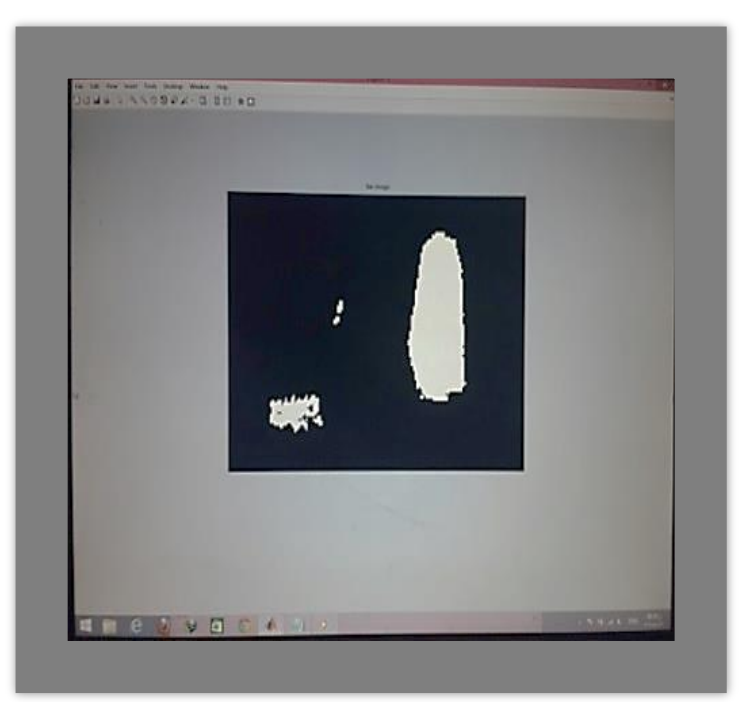

Fig.(3): Binary image.

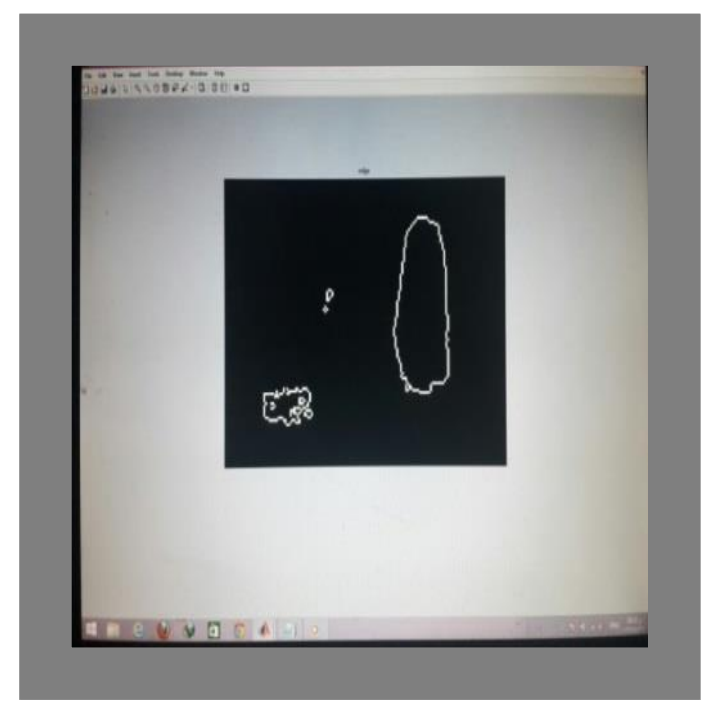

Fig.(4): Edge detection (sobel method).

4) Using the most morphological opration are dilation and fill

- Dilation adds pixels to the boundaries of objects to leave some boundary around obstacles so as avoid the collision between robot and obstacles as shown in Fig.(5), the number of pixels added to object depends on the size and shape of structuring element that it's used to process the image.

s1 = strel ('line', 3,90);

s2 = strel ('line', 3,0);

$\mathrm{e}=$ imdilate (e1, [s1 s2]);

Where strel function used to create any size and shape of structuring elements, It's support for many common shapes such as lines, diamond, disk, periodic and balls.

- Fill used to fill the space of objects in an image as shown in Fig.(6). $\mathrm{f}=$ imfill (e, 'holes');

5) Determine if there is any obstacle in environment by using voronoi scheme as a roadmap, then finding all possible paths for the robot to move to the goal by using distance equation.

\section{Voronoi diagram}

The most important approaches roadmap methods are based on visibility graphs and voronoi diagrams, the voronoi diagram can be very useful in robot path planning if the points represent obstacles in space.

Voronoi diagram is a set of sites in the space or a collection of regions that divide up the space Each region corresponds to one of the sites and all points in one region are closer to the site representing the region than to any other site [7]. A Voronoi diagram is sometimes known as a Dirichlet Tessellation. The cells are called Dirichlet regions or Voronoi cells [8].

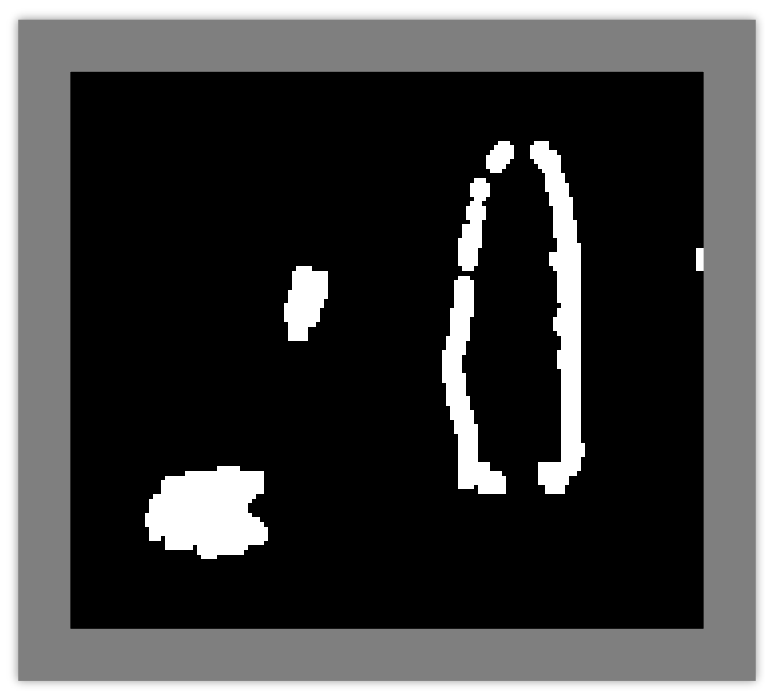

Fig.(5): Morphological operation (imdilate).

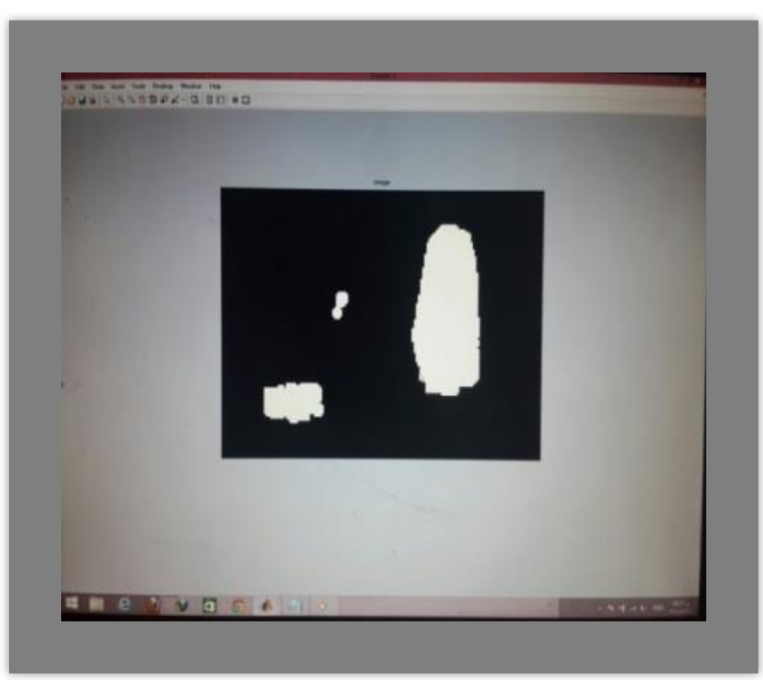

Fig.(6): Morphological operation (imfill). 
Voroni diagrams ere considered as early at 1644 by René Descartes and were used by Dirichlet (1850) in the investigation of positive quadratic forms. They were also studies by Voroni (1970), who extended the investigation of voroni diagrams to higher dimensions. They find wide spread applications in areas such as meteorology, image segmentation optical character recognition and other computation applications. Beside points such diagrams use lines and polygons as sits, by increasing the diagrams with line segments that connect to nearest points on the sites, a space subdivision of the environment is obtained [9].

This structure can be used as an navigation method for finding path through large space. A visibility graph is a graph whose include the start, target and the vertices of polygonal obstacles [10]. It's edges are the edges of the obstacles and edges joining all pairs of vertices that can see each other. After using this method to compute the shortest paths, We found the robot may be touch the obstacles at the vertices or even edges of obstacles as shown in Fig.(7). For this disadvantage, We used another method of roadmap it's voronoi diagrams method as shown in Fig.(8).

Road-Map Path Planning: Visibility Graph



Fig.(7): Path planning by using visibility graph method.

Road-Map Path Planning: Voronoi Diagram

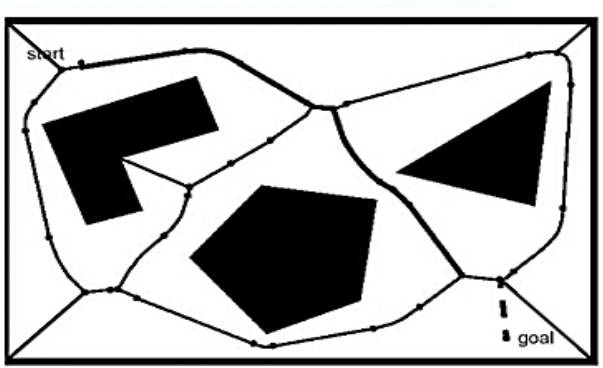

Fig.(8): Path planning by using voronoi diagram method.

\section{Experiment and results}

Path planning is one of the most important elements for mobile robot. Path planning is the determination of a path that a robot must take in order to pass over each point in an environment .path planning problem is how to detect stationary obstacles in environment to find a sequence of valid points that lead from the starting point to the goal, we can achieve this by determine the boundaries polygon into small segments.

The Voronoi diagram computes for this collection of approximating points.

Once this complicated Voronoi diagram is constructed as shown in Fig.(9).We need to find vertices of the Voronoi edges vr and vc, By using distance equation with these points, we can plan from start position to goal position consists of three steps.

Distance equation:

$\mathrm{D}=\sqrt{(v r-x)^{2}+(v c-y)^{2}}$

where $(\mathrm{x}, \mathrm{y})$ represent the start point.

Given a voroni diagram (VDS) planning from start position to goal position consists of three steps.

1. We plan from start point to its closest point on voronoi diagrams (VDS).

2. Plan along the voronoi diagrams (VDS) until the point closest to the goal is reached (the departure point).

3. Plan from the departure point to the goal.

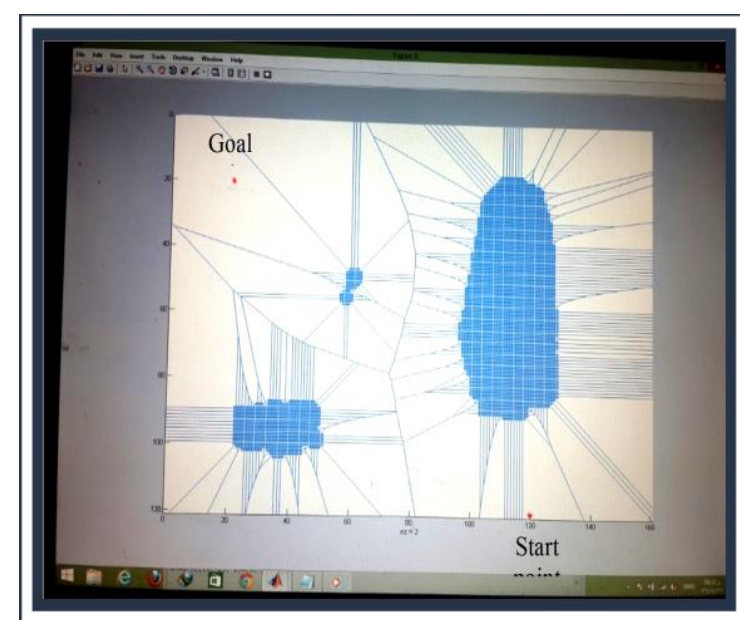

Fig.(9): Voronoi diagram.

The number of objects and the number of paths would vary depending on situation of vision of the robot. After provides all possible paths for the robot, the robot would then be able to choose the shortest path. 
Path 1: it is possible but it isn't the shortest path as shown in Fig.(10).

Path 2: it is possible but it isn't the shortest path as shown in Fig.(11).

Path 3: it is the shortest path and possible as shown in Fig.(12).

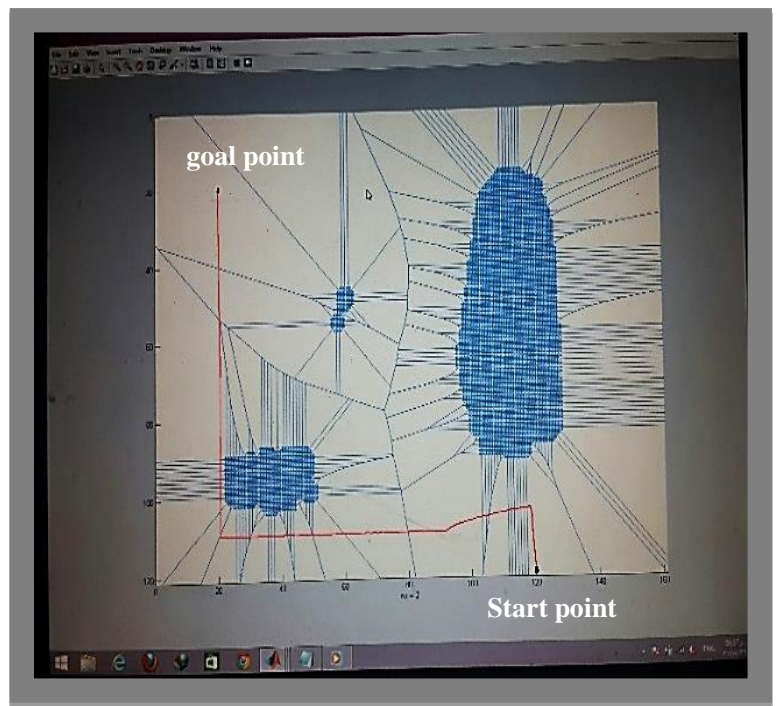

Fig.(10): Path 1.

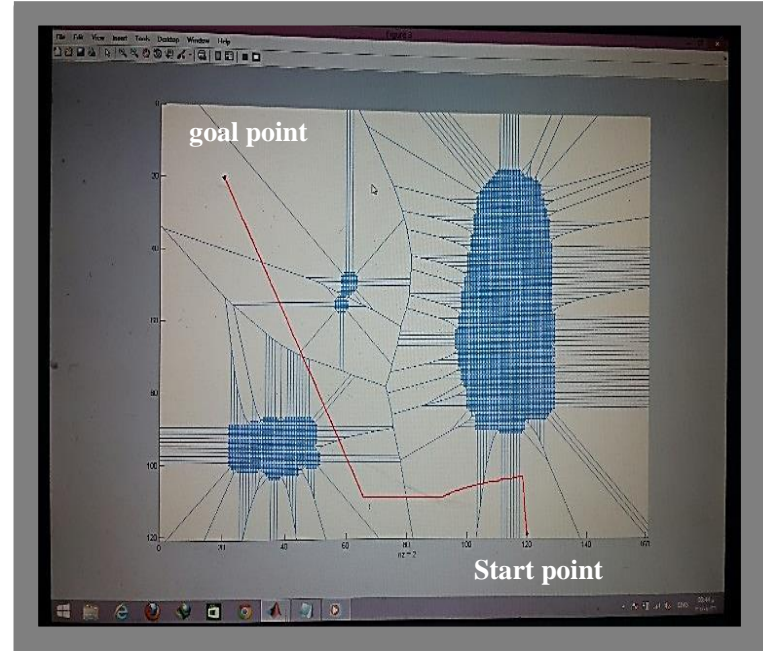

Fig.(11): path 2.

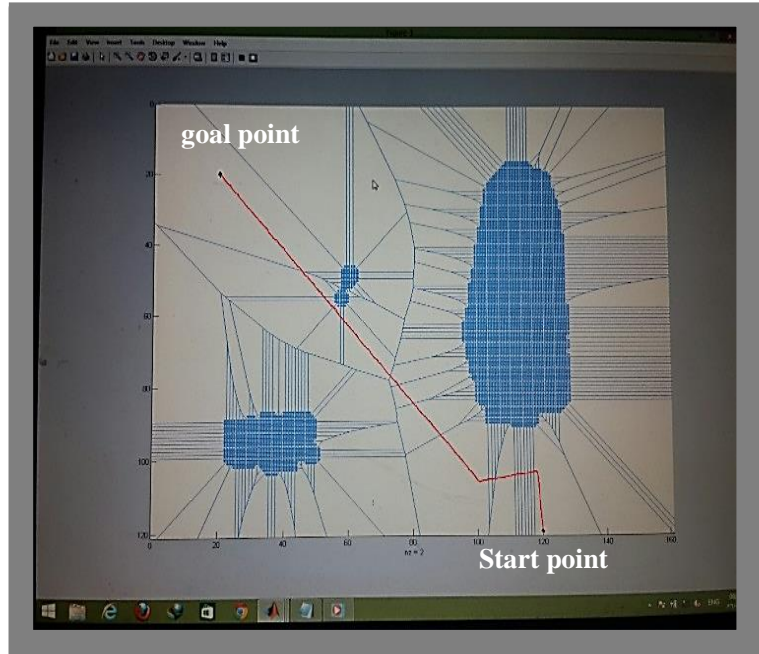

Fig.(12): path 3: the shortest path.

\section{Conclusion}

This paper provides a framework for mobile robot navigation by analysis the vision of robot using laptop camera to capture an image of environment for identified the location of obstacles then, using voronoi diagrams to determine shortest path for the robot to reach his goal.

\section{Future Work}

We present anew intelligent hybrid technique based on the combination of fuzzy inference system and artificial neural network for solving path planning for mobile robot the system would be further developed with moving obstacles and moving target.

\section{References}

[1] Shahed Sh., Sallehuddin M. Haris, Elham G. and Ali Sh., "Webcam-Based Mobile Robot Planning Using Voronoi Diagrams and Image Processing", 9th WSEAS International Conference on Applications of Electrical Engineering, 151-156, 2010.

[2] G. Blanchet, M. Charbit "Digital Signal Image Processing Using Matlab", isteltd, 2006.

[3] Abhishek Ch., Ketki G., Shalaka G., Sumeet A., Mrs. Pooja Kulkarni, "Path Planning For Mobile Robot Navigation Using Image Processing", International Journal of Scientific \& Engineering Research, 4(6), June-2013.

[4] A. Ohya, A. Kak, "Vision - Based Navigation By a Mobile Robot With Obstacle Avoidance Using Single-Camera Vision and Ultrasonic Sensing”, Robotics and Automation, IEEE Transactions, Dec. 1998.

[5] Buniyamin N., Wan Ng., Sariff N., Mohamad Z., "A Simple Local Path Planning Algorithm for Autonomous Mobile Robots", International Journal Of System Application, Engineering \& Development 5(2), 2011.

[6] J. Campbell, R. Sukthankar, Nourbakhsh, I. Sukthankar and A. Pahwa, "A Robust Visual Odometry and Precipice Detection 
System Using Consumer - Grade

Monocular Vision", Proc. ICRA2005,

Barcelona, Spain, 2005.

[7] Franz A., "Voronoi Diagrams - A Survey of a Fundamental Geometric Data Structure", ACM Computing Surveys, 23(3), 345-405, 1991.

[8] Geraerts, Roland, "Planning Short Paths with Clearance Using Explicit”, Corridors (PDF), International Conference on Robotics and Automation, IEEE, 19972004, 2010.

[9] Atsuyuki O., Barry Boots, Kokichi S., Sung Nok Ch., "Spatial Tessellations Concepts and Applications of Voronoi Diagrams", John Wiley, 671, 2000.

[10] A. Okabe, B. Boots, Sugihara K., Chiu, "Spatial Tessellations and Applications of Voronoi Diagrams", John Wiley \& Sons, New York, 2000.



هذا البحث يقدم طريقة لابحار روبوت منتقل في مسار

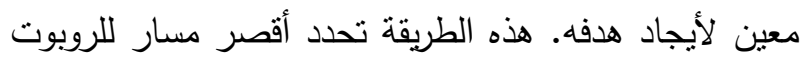

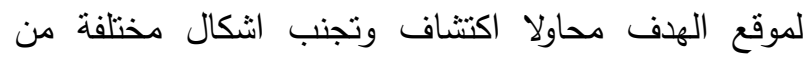

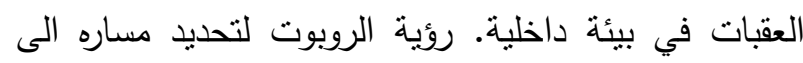

الهدف تلثقط كصورة بأستخدام كاميرا الحاسوب المحمول وينت

تحليل الصورة باستخدام معالجة الصور بلغة الماتلاب لتحديد

موقع العوائق. نظرية فوروني هي طريقة لتحديد جميع لئه

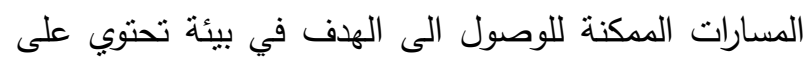

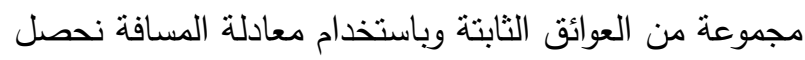

على اقصر مسار للروبوت بين ثلاث مسارات.

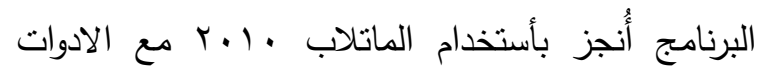

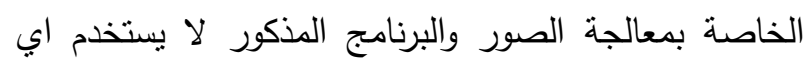

نوع من المتحسسات سوى كاميرا الحاسوب المحمول.
} 